\title{
The 2020 national diagnostic reference levels for nuclear medicine in Japan
}

\author{
Koichiro Abe ${ }^{1}\left(\right.$ D $\cdot$ Makoto Hosono $^{2} \cdot$ Takayuki Igarashi $^{3} \cdot$ Takashi limori $^{4} \cdot$ Masanobu Ishiguro $^{5} \cdot$ Teruo Ito $^{6}$. \\ Tomomasa Nagahata $^{7} \cdot$ Hiroyuki Tsushima $^{8} \cdot$ Hiroshi Watanabe $^{9}$
}

Received: 5 July 2020 / Accepted: 16 August 2020 / Published online: 27 August 2020

(c) The Author(s) 2020

\begin{abstract}
The diagnostic reference levels (DRLs) are one of several effective tools for optimizing nuclear medicine examinations and reducing patient exposure. With the advances of imaging technology and alterations of examination protocols, the DRLs must be reviewed periodically. The first DRLs in Japan were established in 2015, and since 5 years have passed, it is time to review and revise the DRLs. We conducted a survey to investigate the administered activities of radiopharmaceuticals and the radiation doses of computed tomography (CT) in hybrid CT accompanied by single photon emission computed tomography (SPECT)/CT and positron emission tomography (PET)/CT. We distributed a Web-based survey to 915 nuclear medicine facilities throughout Japan and survey responses were provided by 256 nuclear medicine facilities (response rate $28 \%$ ). We asked for the facility's median actual administered activity and median radiation dose of hybrid CT when SPECT/ CT or PET/CT was performed for patients with standard habitus in the standard protocol of the facility for each nuclear medicine examination. We determined the new DRLs based on the 75th percentile referring to the 2015 DRLs, drug package inserts, and updated guidelines. The 2020 DRLs are almost the same as the 2015 DRLs, but for the relatively long-lived radionuclides, the DRLs are set low due to the changes in the Japanese delivery system. There are no items set higher than the previous values. Although the DRLs determined this time are roughly equivalent to the DRLs used in the US, overall they tend to be higher than the European DRLs. The DRLs of the radiation dose of CT in hybrid CT vary widely depending on each imaging site and the purpose of the examination.
\end{abstract}

Keywords Diagnostic reference level $\cdot$ DRL $\cdot$ Nuclear medicine $\cdot$ Radiopharmaceutical $\cdot$ Hybrid CT

\section{Background}

The use of radiation in the medical field continues to expand. Although patients who are exposed to radiation during their diagnoses and treatment often have the tremendous opportunity to overcome life-threatening illnesses, they are also at increased risk of radiation damage. Radiation-based diagnoses and treatment are justified only when the benefit is clearly greater than the risk associated with the radiation exposure (justification), and it is necessary to take measures

Koichiro Abe, Makoto Hosono, Takayuki Igarashi, Takashi Iimori, Masanobu Ishiguro, Teruo Ito, Tomomasa Nagahata, Hiroyuki Tsushima and Hiroshi Watanabe made equal contributions to this work.

Koichiro Abe

k-abe@tokyo-med.ac.jp

Extended author information available on the last page of the article to reduce radiation doses as much as possible during justified radiological diagnoses and treatment (optimization) [1]. The motivation to reduce and manage patients' radiation exposure as much as possible while maintaining the quality of radiation medicine is increasing worldwide [2].

In a survey conducted in the mid-1980s, British researchers reported that there were up to 50-fold differences in institutional radiation exposures to patients in domestic X-ray examinations [3], and they advocated the concept of guideline reference doses derived from patient-exposure doses in routine X-ray examinations. Each hospital was then able to compare its own doses with the guideline reference doses and identify the radiation exposure doses for which efforts could be most efficiently directed towards minimizing the patient dose. The concept of guideline reference doses was later established by the International Commission on Radiologic Protection (ICRP) [4-7] and the International Atomic Energy Agency 
(IAEA) [8]. With the progress of radiation generators and computer technology, a reference dose was set for each imaging modality, and these reference doses have become widely known as the diagnostic reference levels (DRLs) today.

The DRLs are determined by the following procedure: First, the most common protocol for each type of radiological examination performed at each institution is identified. Second, the median radiation exposure dose for $\geq 20$ standard-size patients in the protocol is calculated. Third, the 75th percentile of the distribution of studied institutions for the median radiation exposure dose value is determined. Finally, the DRLs are established based on the 75th percentile [9]. In case the values thus derived were considered unreliable or inappropriate, the DRLs in the present study were established either by referring to the current DRLs, the drug package inserts or guidelines. Since the performance of radiological diagnostic equipment and the protocol of each radiological examination would vary depending on regional conditions and the era, DRLs should be established for each country or region, and should be reviewed periodically.

\section{Initiatives in Japan}

The Japan Network for Research and Information on Medical Exposures (J-RIME) was established in 2010 to collect and share information on medical exposure studies and to discuss and implement measures for cooperation with international organizations. One of the first major projects of the J-RIME was the setting of DRLs, which provide a tool for the above-mentioned optimization. The J-RIME has collaborated with related societies and associations including the Japanese Society of Nuclear Medicine (JSNM) and the Japanese Society of Nuclear Medicine Technology (JSNMT). The J-RIME conducted a nationwide survey on the actual administered radioactivity in adults, and it established Japan's national DRLs in nuclear medicine in 2015 [10].

Because 5 years have already passed since the first DRLs were set, it is time to review the DRLs in light of the emergence of new drugs and the development of new technologies. Over the last 5 years, radiopharmaceuticals including ${ }^{111}$ In-pentetreotide were newly approved for use in Japan, and they were added as new items to the DRLs. Conversely, as the clinical uses of ${ }^{133} \mathrm{Xe}$ gas, ${ }^{99 \mathrm{~m}} \mathrm{Tc}-\mathrm{HSA}$, and ${ }^{131} \mathrm{I}$-MIBG have been discontinued in Japan, these items were deleted from the DRLs. As the uses of single photon emission computed tomography (SPECT)/computed tomography (CT) and positron emission tomography (PET)/CT have become widespread, the radiation exposure doses provided by hybrid CT are also included in the 2020 DRLs.

\section{Nationwide survey targeting facilities, period, and method}

The targets of the survey that we carried out were the hospitals, clinics, imaging centers, and other facilities that carry out nuclear medicine examinations across Japan. We created a survey form on the Web and asked each facility to enter its response to each item. The survey concerned the facility's nuclear medicine examinations conducted in July 2019, and each facility input on the Web the median of the actual administered activities of radiopharmaceuticals at their facility, plus the computed tomography dose index volume (CTDIvol) and dose length product (DLP) when hybrid CT was performed. The survey input period was from August 26, 2019 to September 24, 2019.

This work was exempted from an ethical review because (1) the recording and management of patient exposure are mandatory in nuclear medicine and some other radiological modalities in accord with provisions of the Ordinance for Enforcement of the Medical Care Act, and (2) this work collected and analyzed only median values of each institution from its existing data, without handling information of each patient or examination.

\section{How the new DRLs were determined}

We asked 915 nuclear medicine facilities throughout Japan to complete a Web-based questionnaire, and 256 of the facilities entered their answers on the Web (response rate $28 \%)$.

We determined the new DRLs in nuclear medicine based on the following principles.

1. For radiopharmaceuticals, we asked each facility to enter the median actual administered activity of radioisotope (RI) for each nuclear medicine examination conducted for standard-size patients. This survey was conducted for adults only; for children aged $\leq 15$ years, the consensus guidelines for pediatric nuclear medicine in Japan were developed by the JSNM in 2014 [11].

2. For the radiation doses of hybrid CT of SPECT/CT or PET/CT, we asked each facility to enter the median CTDIvol and DLP values for each nuclear medicine examination conducted for standard-size adults. For children aged $\leq 15$ years, the CTDIvols and the DLPs were published as part of Japan's National 2020 DRLs [12].

3. The entered values were aggregated for type of nuclear medicine examination, and outliers that were apparently mistaken for input were excluded. 
4. Regarding the administered activities of radiopharmaceuticals and the irradiation doses of hybrid CT, we calculated the 50th and 75th percentiles from the distributions of the values input by each facility.

5. For radiopharmaceuticals, for which values thus derived were considered unreliable or inappropriate, we determined the 2020 DRLs either by referring to the 2015 DRLs, drug package inserts, or current societal practice guidelines.

6. Finally, a reasonable value based on the 75th percentile was set as a two-digit integer value in principle.

\section{DRLs for administered activities of radiopharmaceuticals}

The 2020 DRLs were determined as described above. For some items such as O-gas and amyloid PET, where the numbers of responses were $<10$, we used the values shown in the 2015 DRLs or the drug package inserts as-is.

The survey results (50th and 75th percentiles), the determined 2020 DRLs, and the 2015 DRLs for each item are shown in Table 1 (radiopharmaceuticals for scintigraphy) and Table 2 (radiopharmaceuticals for PET). The radiopharmaceuticals or examination items that were newly added between the 2015 DRLs and the 2020 DRLs are ${ }^{99 \mathrm{~m}}$ Tc-HSA$\mathrm{D}$ for protein-losing gastroenteropathy, ${ }^{111} \mathrm{In}$-pentetreotide for somatostatin receptor imaging, ${ }^{18}$ F-FDG for aortitis, and amyloid tracers. The former three tracers were approved for Health Care Insurance coverage in Japan over the past 5 years. Because the three amyloid tracers were licensed for clinical use but not yet covered by insurance, examinations using these tracers were rarely performed and the number of survey responses was very small. There was no response from any facility about ${ }^{18} \mathrm{~F}$-florbetaben. The DRLs for the amyloid tracers other than ${ }^{18} \mathrm{~F}$-flutemetamol were thus determined based on the drug package inserts.

Similar to the previous survey, we observed a tendency among the majority of nuclear medicine facilities; these facilities have been administering radiopharmaceutical activities based on the drug package inserts and guidelines, and many items in the 2020 DRLs are set equal to or lower than those in the 2015 DRLs. There are no items set higher than the previous values. Although this might be due to the advances in imaging devices and image reconstruction technology that have been obtained over the last five years, it is hoped that this is because the optimization of radiopharmaceutical use at each facility has been widely promoted by the establishment of the national DRLs.

Among the radiopharmaceuticals, the activity of ${ }^{111}$ In-chloride for bone marrow scintigraphy has largely decreased from $120 \mathrm{MBq}$ in the 2015 DRLs to $80 \mathrm{MBq}$ in the 2020 DRLs (a 33\% decrease). Similarly, the activities of ${ }^{111} \mathrm{In}$-DTPA for cisternography, ${ }^{201} \mathrm{Tl}$ scintigraphy for myocardium and tumor, and ${ }^{67} \mathrm{Ga}$ scintigraphy for tumor or inflammation have decreased from $70 \mathrm{MBq}$ in the 2015 DRLs to $40 \mathrm{MBq}$ in the 2020 DRLs (a $43 \%$ decrease), from 180 to $120 \mathrm{MBq}$ (a 33\% decrease), and from 200 to $120 \mathrm{MBq}$ (a 40\% decrease), respectively. The main reasons for these decreases is likely to be the modifications of delivery systems made by manufacturers in 2016. Before then, Japanese manufacturers delivered radiopharmaceuticals with relatively long half-life nuclides such as ${ }^{111} \mathrm{In},{ }^{201} \mathrm{Tl}$, and ${ }^{67} \mathrm{Ga}$ a few days before the assay date. In many nuclear medicine facilities, the pharmaceuticals containing these RIs used to be administered without activity modification on the day they were delivered, resulting in administration activities that were higher than the assay activities. After 2016, as these pharmaceuticals are now delivered on the assay day, the latest DRLs are lower than the 2015 DRLs.

The DRLs that we determined herein are roughly equivalent to the US DRLs with the following exceptions: higher ${ }^{99 \mathrm{~m}}$ Tc-MAA (260 vs. $215 \mathrm{MBq}$ ) and lower ${ }^{111}$ In-pentetreotide (120 vs. $237 \mathrm{MBq}$ ) and lower ${ }^{18} \mathrm{~F}-\mathrm{FDG}$ (240 vs. $555 \mathrm{MBq}$ for tumor, and 240 vs. $414 \mathrm{MBq}$ for brain) [13]. The European DRLs are generally lower than those of the US and Japan [14]. Although differences in these DRLs can probably be attributed to differences in national/regional development status, customs, patient habitus and weight, etc., it might be necessary for each country/region to examine whether a given activity can be reduced with preserved image quality and whether nuclear medicine examinations have sufficient image quality with the currently used administration activities.

\section{DRLs for CT doses in hybrid CT}

We also investigated hybrid CT examinations, i.e., SPECT/ CT (Table 3) and PET/CT (Table 4) which were not examined in the previous survey, and we determined the DRLs of the CT irradiation dose. For the dose evaluation, we used the CTDIvol (mGy) and DLP (mGy $\mathrm{cm}$ ) displayed on the CT console or recorded in the dose report. We prepared survey question for seven body regions (the whole body, brain, head and neck, chest, heart, abdomen and pelvis, and extremities) and for the examinations' purposes, e.g., attenuation correction (AC) only and $\mathrm{AC}+$ diagnosis (Dx) for SPECT/CT (Table 3). Compared to radiological diagnostic CT, SPECT/ $\mathrm{CT}$ is still much less frequently performed at present, and $\mathrm{CT}$ is rarely performed for the purpose of $\mathrm{AC}$ alone even in brain and cardiac PET scans in Japan. In fact, there were only a few survey responses about brain and cardiac PET/ $\mathrm{CT}$ in which CT was used for AC only. Regarding PET/CT, we asked CT dose for each three body region (whole body, brain, and heart), and the questions were divided into two 
Table 1 Survey results and DRLs: radiopharmaceuticals for scintigraphy

\begin{tabular}{|c|c|c|c|c|}
\hline \multirow[t]{2}{*}{ Procedure and radiopharmaceutical } & \multicolumn{2}{|c|}{ Median activity of radiopharmaceutical, $\mathrm{MBq}$} & \multirow{2}{*}{$\begin{array}{l}2020 \text { DRLs, } \\
\mathrm{MBq}\end{array}$} & \multirow{2}{*}{$\begin{array}{l}2015 \\
\text { DRLs, } \\
\text { MBq }\end{array}$} \\
\hline & 50th percentile & 75th percentile & & \\
\hline Bone: ${ }^{99 \mathrm{~m}} \mathrm{Tc}-\mathrm{MDP}$ & 879.9 & 951.2 & 950 & 950 \\
\hline Bone: ${ }^{99 \mathrm{~m}} \mathrm{Tc}-\mathrm{HMDP}$ & 882.9 & 986.0 & 950 & 950 \\
\hline Bone marrow: ${ }^{111}$ In-chloride & 76.0 & 76.9 & 80 & 120 \\
\hline Cerebral blood flow: ${ }^{99 \mathrm{~m}} \mathrm{Tc}-\mathrm{HM}-\mathrm{PAO}$, rest or stress & 760.0 & 871.1 & 800 & 800 \\
\hline Cerebral blood flow: ${ }^{99 \mathrm{~m}} \mathrm{Tc}-\mathrm{HM}-\mathrm{PAO}$, rest and stress & 1110.0 & 1116.0 & 1200 & 1200 \\
\hline Cerebral blood flow: ${ }^{99 \mathrm{~m}} \mathrm{Tc}-\mathrm{ECD}$, rest or stress & 755.0 & 796.5 & 800 & 800 \\
\hline Cerebral blood flow: ${ }^{99 \mathrm{~m}} \mathrm{Tc}-\mathrm{ECD}$, rest and stress & 1000.0 & 1088.0 & 1100 & 1100 \\
\hline Cerebral blood flow: ${ }^{123}$ I-IMP, rest or stress & 183.0 & 206.1 & 200 & 200 \\
\hline Cerebral blood flow: ${ }^{123}$ I-IMP, rest and stress & 249.8 & 272.0 & 270 & 300 \\
\hline Benzodiazepine receptor: ${ }^{123}$ I-iomazenil & 185.0 & 195.5 & 200 & 200 \\
\hline Dopamine transporter: ${ }^{123}$ I-ioflupane & 184.5 & 190.3 & 190 & 190 \\
\hline Cisternography: ${ }^{111}$ In-DTPA & 38.0 & 38.2 & 40 & 70 \\
\hline Thyroid imaging: $\mathrm{Na}^{123} \mathrm{I}$ & 8.1 & 8.7 & 10 & 10 \\
\hline Thyroid imaging: ${ }^{99 \mathrm{~m}} \mathrm{Tc}-\mathrm{O}_{4}{ }^{-}$ & 185.0 & 238.5 & 240 & 300 \\
\hline Parathyroid: ${ }^{201}$ Tl- chloride & 75.7 & 111.6 & 120 & 120 \\
\hline Parathyroid: ${ }^{99 \mathrm{~m}} \mathrm{Tc}-\mathrm{O}_{4}{ }^{-}$ & 224.0 & 370.0 & 300 & 300 \\
\hline Parathyroid: ${ }^{99 \mathrm{~m}} \mathrm{Tc}-\mathrm{MIBI}$ & 745.5 & 828.1 & 800 & 800 \\
\hline Lung ventilation: ${ }^{81 \mathrm{~m}} \mathrm{Kr}$-gas & 185.0 & 190.6 & 200 & 200 \\
\hline Lung perfusion: ${ }^{99 \mathrm{~m}} \mathrm{Tc}-\mathrm{MAA}$ & 201.0 & 280.0 & 260 & 260 \\
\hline Venography: ${ }^{99 \mathrm{~m}} \mathrm{Tc}-\mathrm{MAA}$ & 370.0 & 477.5 & 500 & 500 \\
\hline Liver and spleen: ${ }^{99 \mathrm{~m}} \mathrm{Tc}$-phytate & 185.0 & 200.0 & 200 & 200 \\
\hline Liver function: ${ }^{99 \mathrm{~m}} \mathrm{Tc}-\mathrm{GSA}$ & 233.0 & 260.0 & 260 & 260 \\
\hline Hepatobiliary: ${ }^{99 \mathrm{~m}} \mathrm{Tc}-\mathrm{PMT}$ & 233.0 & 260.0 & 260 & 260 \\
\hline Liver and spleen: ${ }^{99 \mathrm{~m}} \mathrm{Tc}-\mathrm{Sn}$ colloid & 144.5 & 185.0 & 180 & 180 \\
\hline Myocardial perfusion: ${ }^{201} \mathrm{Tl}$ - chloride & 113.0 & 114.0 & 120 & 180 \\
\hline Myocardial perfusion: ${ }^{99 \mathrm{~m}} \mathrm{Tc}$-tetrofosmin, rest or stress & 740.0 & 836.0 & 840 & 900 \\
\hline Myocardial perfusion: ${ }^{99 \mathrm{~m}} \mathrm{Tc}$-tetrofosmin, rest and stress & 1077.2 & 1200.0 & 1200 & 1200 \\
\hline Myocardial perfusion: ${ }^{99 \mathrm{~m}} \mathrm{Tc}-\mathrm{MIBI}$, rest or stress & 755.2 & 874.6 & 880 & 900 \\
\hline Myocardial perfusion: ${ }^{99 \mathrm{~m}} \mathrm{Tc}-\mathrm{MIBI}$, rest and stress & 1076.4 & 1229.0 & 1200 & 1200 \\
\hline Myocardial fatty acid metabolism: ${ }^{123}$ I-BMIPP & 124.0 & 129.1 & 130 & 130 \\
\hline Cardiac sympathetic nerve imaging: ${ }^{123}$ I-MIBG & 126.0 & 129.9 & 130 & 130 \\
\hline Cardiac blood pool: ${ }^{99 \mathrm{~m}} \mathrm{Tc}-\mathrm{HSA}-\mathrm{D}$ & 835.0 & 966.0 & 970 & 1000 \\
\hline Myocardial infarction: ${ }^{99 \mathrm{~m}} \mathrm{Tc}-\mathrm{PYP}$ & 740.0 & 809.5 & 800 & 800 \\
\hline Salivary gland: ${ }^{99 \mathrm{~m}} \mathrm{Tc}-\mathrm{O}_{4}{ }^{-}$ & 209.4 & 370.0 & 370 & 370 \\
\hline Meckel's diverticulum: ${ }^{99 \mathrm{~m}} \mathrm{Tc}-\mathrm{O}_{4}{ }^{-}$ & 370.0 & 432.8 & 440 & 500 \\
\hline Gastrointestinal bleeding: ${ }^{99 \mathrm{~m}}$ Tc-HSA-D & 933.0 & 1045.0 & 1040 & 1040 \\
\hline Protein losing gastroenteropathy: ${ }^{99 \mathrm{~m}} \mathrm{Tc}-\mathrm{HSA}-\mathrm{D}$ & 932.0 & 1041.0 & 1040 & - \\
\hline Renal imaging, static: ${ }^{99 \mathrm{~m}} \mathrm{Tc}-\mathrm{DMSA}$ & 185.0 & 212.8 & 210 & 210 \\
\hline Renal imaging, dynamic: ${ }^{99 \mathrm{~m}} \mathrm{Tc}-\mathrm{MAG}_{3}$ & 286.5 & 375.6 & 380 & 400 \\
\hline Renal imaging, dynamic: ${ }^{99 \mathrm{~m}} \mathrm{Tc}-\mathrm{DTPA}$ & 356.5 & 390.3 & 390 & 400 \\
\hline Adrenal cortex: ${ }^{131}$ I-Adosterol & 37.0 & 39.4 & 40 & 44 \\
\hline Adrenal medulla: ${ }^{123}$ I-MIBG & 128.1 & 132.4 & 130 & 130 \\
\hline Tumor: ${ }^{201}$ Tl-chloride & 113.9 & 114.2 & 120 & 180 \\
\hline Tumor and inflammation: ${ }^{67} \mathrm{Ga}$-citrate & 113.0 & 114.0 & 120 & 200 \\
\hline Somatostatin receptor: ${ }^{111}$ In-pentetreotide & 156.0 & 185.7 & 120 & - \\
\hline Lymphatic system: ${ }^{99 \mathrm{~m}} \mathrm{Tc}-\mathrm{HSA}-\mathrm{D}$ & 440.0 & 830.0 & 830 & 950 \\
\hline Sentinel lymph node, breast cancer: ${ }^{99 \mathrm{~m}} \mathrm{Tc}$ colloid & 74.0 & 111.0 & 120 & 120 \\
\hline Sentinel lymph node, breast cancer: ${ }^{99 \mathrm{~m}} \mathrm{Tc}-$ phytate & 72.8 & 100.0 & 120 & 120 \\
\hline
\end{tabular}


Table 1 (continued)

\begin{tabular}{|c|c|c|c|c|}
\hline \multirow[t]{2}{*}{ Procedure and radiopharmaceutical } & \multicolumn{2}{|c|}{ Median activity of radiopharmaceutical, $\mathrm{MBq}$} & \multirow{2}{*}{$\begin{array}{l}2020 \text { DRLs, } \\
\mathrm{MBq}\end{array}$} & \multirow{2}{*}{$\begin{array}{l}2015 \\
\text { DRLs, } \\
\mathrm{MBq}\end{array}$} \\
\hline & 50th percentile & 75th percentile & & \\
\hline Sentinel lymph node, melanoma: ${ }^{99 \mathrm{~m}} \mathrm{Tc}$ colloid & 92.5 & 152.0 & 120 & 120 \\
\hline Sentinel lymph node, melanoma: ${ }^{99 \mathrm{~m}} \mathrm{Tc}$-phytate & 78.0 & 111.0 & 120 & 120 \\
\hline RI angiography: ${ }^{99 m} \mathrm{Tc}-\mathrm{HSA}-\mathrm{D}$ & 932.0 & 1045.0 & 1000 & 1000 \\
\hline
\end{tabular}

Table 2 Survey results and DRLs: radiopharmaceuticals for PET

\begin{tabular}{|c|c|c|c|c|}
\hline \multirow[t]{2}{*}{ Procedure and radiopharmaceutical } & \multicolumn{2}{|c|}{$\begin{array}{l}\text { Median activity of radiopharmaceutical, } \\
\text { MBq }\end{array}$} & \multirow[t]{2}{*}{2020 DRLs, MBq } & \multirow{2}{*}{$\begin{array}{l}2015 \\
\text { DRLs, } \\
\text { MBq }\end{array}$} \\
\hline & 50th percentile & 75th percentile & & \\
\hline Tumor: ${ }^{18} \mathrm{~F}-\mathrm{FDG}$, in-house-produced & 220.2 & 235.1 & 240 & 240 \\
\hline Tumor: ${ }^{18} \mathrm{~F}-\mathrm{FDG}$, delivery & 257.0 & 286.5 & 240 & 240 \\
\hline Tumor: ${ }^{18} \mathrm{~F}-\mathrm{FDG}$, per body weight & 3.7 & 4.0 & 4 & - \\
\hline Aortitis: ${ }^{18} \mathrm{~F}-\mathrm{FDG}$, in-house-produced & 238.0 & 254.5 & 240 & - \\
\hline Aortitis: ${ }^{18} \mathrm{~F}-\mathrm{FDG}$, delivery & 249.5 & 276.9 & 240 & - \\
\hline Aortitis: ${ }^{18}$ F-FDG, per body weight & 3.7 & 4.0 & 4 & - \\
\hline Myocardial metabolism: ${ }^{18} \mathrm{~F}-\mathrm{FDG}$, in-house-produced & 226.0 & 259.0 & 240 & 240 \\
\hline Myocardial metabolism: ${ }^{18} \mathrm{~F}-\mathrm{FDG}$, delivery & 259.0 & 322.1 & 240 & 240 \\
\hline Myocardial metabolism: ${ }^{18} \mathrm{~F}-\mathrm{FDG}$, per body weight & 3.7 & 4.9 & 5 & - \\
\hline Myocardial perfusion: ${ }^{13} \mathrm{~N}-\mathrm{NH}_{3}$ & 428.1 & 524.2 & 520 & 720 \\
\hline Brain: ${ }^{18} \mathrm{~F}$-FDG, in-house-produced & 196.0 & 223.2 & 240 & 240 \\
\hline Brain: ${ }^{18} \mathrm{~F}-\mathrm{FDG}$, delivery & 230.0 & 254.9 & 240 & 240 \\
\hline Brain: ${ }^{18}$ F-FDG, per body weight & 3.7 & 3.7 & 4 & - \\
\hline Amyloid: ${ }^{18}$ F-flutemetamol, in-house-produced & 184.8 & 184.9 & $260^{\mathrm{a}}$ & - \\
\hline Amyloid: ${ }^{18}$ F-flutemetamol, delivery & 209.0 & 260.8 & 260 & - \\
\hline Amyloid: ${ }^{18} \mathrm{~F}$-florbetapir, in-house-produced & 370.0 & 370.0 & $370^{\mathrm{b}}$ & - \\
\hline Amyloid: ${ }^{18} \mathrm{~F}$ - florbetapir, delivery & 370.0 & 373.5 & $370^{\mathrm{b}}$ & - \\
\hline Amyloid: ${ }^{18} \mathrm{~F}$-florbetaben, in-house-produced & - & - & $300^{\mathrm{b}}$ & - \\
\hline${ }^{15} \mathrm{O}-\mathrm{CO}_{2}$ gas: $2 \mathrm{D}$ & 270.0 & 270.0 & $8000^{c}$ & 8000 \\
\hline${ }^{15} \mathrm{O}-\mathrm{O}_{2}$ gas: $2 \mathrm{D}$ & 390.0 & 390.0 & $6000^{\mathrm{c}}$ & 6000 \\
\hline${ }^{15} \mathrm{O}-\mathrm{CO}$ gas: $2 \mathrm{D}$ & 385.0 & 385.0 & $3000^{c}$ & 3000 \\
\hline${ }^{15} \mathrm{O}-\mathrm{CO}_{2}$ gas: $3 \mathrm{D}$ & 1500.0 & 1800.0 & $1800^{c}$ & 2900 \\
\hline${ }^{15} \mathrm{O}-\mathrm{O}_{2}$ gas: $3 \mathrm{D}$ & 2000.0 & 4500.0 & $4500^{c}$ & 7000 \\
\hline${ }^{15} \mathrm{O}-\mathrm{CO}$ gas: $3 \mathrm{D}$ & 3000.0 & 3600.0 & $3600^{c}$ & 7500 \\
\hline
\end{tabular}

${ }^{\mathrm{a}}$ The DRL was determined to be same as in-house-produced ${ }^{18} \mathrm{~F}$-flutemetamol

${ }^{\mathrm{b}}$ DRLs were determined based on drug package inserts

${ }^{\mathrm{c}}$ The 2015 DRLs were used as they were

purposes: clinical diagnosis (cl Dx) and cancer screening (Table 4).

The path for establishing the DRLs of hybrid CT used in SPECT/CT and PET/CT is more complicated than that for establishing the DRLs of radiological diagnostic CT. In nuclear medicine, the aspects of an imaging protocol such as the imaged organ, imaged range, number of times of imaging, the purpose of the imaging, etc. differ depending on the type of examination, and the dose of CT exposure varies accordingly. In the UK, the Institute of Physics and
Engineering in Medicine Working Party proposed national DRLs for hybrid CT that were determined for each examination type and purpose of CT [15]. Gardner et al. have developed four distinct dose mode ("low" for attenuation correction, "moderate" for localization, "standard" as diagnosis, and "metal" for orthopedic implants containing metal) based on scan purpose [16]. There is still room for further considerations of how to classify the DRLs for hybrid CT.

A systematic review published in 2018 introduced 14 articles for determining the DRLs for PET/CT and SPECT/ 
Table 3 Survey results and DRLs: hybrid CT of SPECT/CT

\begin{tabular}{|c|c|c|c|c|c|c|}
\hline \multirow[t]{2}{*}{ Target site, purpose of use } & \multicolumn{3}{|l|}{ CTDIvol, mGy } & \multicolumn{3}{|l|}{ DLP, mGy cm } \\
\hline & 50th percentile & 75th percentile & 2020 DRLs & 50th percentile & 75th percentile & 2020 DRLs \\
\hline Whole body, $\mathrm{AC}+\mathrm{Dx}$ & 2.9 & 5.03 & 5.0 & 180 & 384.1 & 380 \\
\hline Brain, $\mathrm{AC}+\mathrm{Dx}$ & 10.6 & 22.60 & 23.0 & 262 & 419.2 & 410 \\
\hline Head and neck, AC + Dx & 3.3 & 5.73 & 5.8 & 133 & 205.1 & 210 \\
\hline Chest, $\mathrm{AC}+\mathrm{Dx}$ & 2.6 & 4.06 & 4.1 & 90 & 164.8 & 170 \\
\hline Heart, $A C+D x$ & 3.2 & 4.50 & 4.5 & 89 & 180.0 & 180 \\
\hline Abdomen, $\mathrm{AC}+\mathrm{Dx}$ & 3.0 & 5.02 & 5.0 & 89 & 206.8 & 210 \\
\hline Extremities, AC + Dx & 3.2 & 4.69 & 4.6 & 159 & 237.2 & 230 \\
\hline Brain, AC only & 7.3 & 12.86 & 13.0 & 140 & 350.9 & 330 \\
\hline Heart, AC only & 1.6 & 4.04 & 4.1 & 45 & 84.1 & 85 \\
\hline
\end{tabular}

Table 4 Survey results and DRLs: hybrid CT of PET/CT

\begin{tabular}{|c|c|c|c|c|c|c|}
\hline \multirow[t]{2}{*}{ Target site, purpose of use } & \multicolumn{3}{|l|}{ CTDIvol, mGy } & \multicolumn{3}{|l|}{ DLP, mGy cm } \\
\hline & 50th percentile & 75th percentile & 2020 DRLs & 50th percentile & 75th percentile & 2020 DRLs \\
\hline Whole body, cl Dx & 4.2 & 6.02 & 6.1 & 451 & 608.6 & 600 \\
\hline Brain, cl Dx & 19.6 & 30.75 & 31.0 & 384 & 641.3 & 640 \\
\hline Heart, cl Dx & 5.5 & 9.03 & 9.1 & 209 & 388.9 & 380 \\
\hline Whole body, cancer screening & 3.5 & 5.50 & 5.5 & 337 & 550.0 & 550 \\
\hline
\end{tabular}

CT [17]. Among 14 PET/CT and five SPECT/CT articles, five PET/CT and two SPECT/CT studies determined the DRLs for hybrid CTs. The values of CTDIvol and DLP varied widely as follows. For PET/CT: CTDIvol 4.3-10.2 mGy and DLP 400-750 mGy cm; for SPECT/CT: CTDIvol 2.1-5.9 mGy of CTDIvols and DLP 36-240 mGy cm. Compared to these values, the CTDIvol and DLP for PET/CT obtained in the present survey are similar at 5.5-31.0 $\mathrm{mGy}$ and 380-640 mGy cm, respectively. However, these values for SPECT/CT in the present survey tend to be high at CTDIvol 4.1-23.0 $\mathrm{mGy}$ and DLP 85-380 $\mathrm{mGy} \mathrm{cm}$. As mentioned above, it seems that these differences are due largely to the differences in CT devices and protocols. We look forward to an opportunity to further reduce patient radiation exposure by comparing other DRLs with a detailed consideration of imaging protocols.

\section{Limitations}

One of the limitations of this study is that there were items for which very few survey responses were obtained. The ICRP recommends using the data for $\geq 10-20$ facilities for the establishment of local DRLs [5]. Because responses from only a few nuclear medicine facilities were available, we determined the DRLs for amyloid and O-gas PET based on the drug package inserts and the 2015 DRLs. Moreover, the survey excluded items with $\leq 10$ responses, resulting in the removal of most of the special-purpose items of hybrid CT. Another limitation is that the survey did not take into account the manufacturing date of the imaging device or the accompanying new technologies. Current advanced techniques, such as semiconductor cameras, time-of-flight, and point spread function technologies, etc. used in SPECT and/or PET, plus the automatic tube current modulation technique, iterative image reconstruction algorithms, and more used in CT can significantly reduce patients' radiation exposure without deterioration of the image quality. Whether each facility adopts these technologies would have a significant impact on the patient exposures.

\section{Future outlook for DRLs}

Although DRLs are becoming an indispensable index for optimizing examination protocols and reducing patient radiation exposure, problems concerning their uses and limitations have been pointed out. The achievable dose (AD) corresponding to the 50th percentile of the used dose has been recommended for use as an effective tool for advancing optimization while ensuring image quality [18]. The administered activity duration product (ADP), which is the product of the administration activity $(\mathrm{MBq})$ and the acquisition time (min), has been proposed as a better measure for optimization with preservation of the image quality $[17,18]$. Rehani proposed a new indicator called the acceptable quality dose 
(AQD), which takes both image quality and patient size into consideration [19]]. We would like to further promote the use of DRLs while utilizing and incorporating these concepts, all of which are designed to reduce or limit patients' radiation exposure.

Acknowledgements This study was performed with financial support from the JSNM and was carried out by the J-RIME, JSNM, JSNMT, Japan Association of Radiological Technologists (JART), Japan Society of Medical Physics (JSMP), Japan Association of Radiological Protection in Medicine (JARPM), Japanese Society of Radiological Technology (JSRT), and Japan Radiological Society (JRS). Part of this study was presented at the JSNM/JSNMT Joint Symposium at the 59th Annual Meeting of the JSNM on November 1, 2019. This work was supported by Health Labour Sciences Research Grant (19IA1004). The authors have no potential conflicts of interest to report.

Open Access This article is licensed under a Creative Commons Attribution 4.0 International License, which permits use, sharing, adaptation, distribution and reproduction in any medium or format, as long as you give appropriate credit to the original author(s) and the source, provide a link to the Creative Commons licence, and indicate if changes were made. The images or other third party material in this article are included in the article's Creative Commons licence, unless indicated otherwise in a credit line to the material. If material is not included in the article's Creative Commons licence and your intended use is not permitted by statutory regulation or exceeds the permitted use, you will need to obtain permission directly from the copyright holder. To view a copy of this licence, visit http://creativecommons.org/licenses/by/4.0/.

\section{References}

1. International Commission on Radiological Protection (ICRP). Recommendations of the International Commission on Radiological Protection. ICRP publication 26. Ann ICRP. 1977;1(3):1-80.

2. Rehani MM, Kushi JF. A study of smart card for radiation exposure history of patient. Am J Roentgenol. 2013;200:780-2.

3. Shrimpton PC, Wall BF, Jones DG, Fisher ES, Hillier MC, Kendall GM, Harrison RM. A national survey of doses to patients undergoing a selection of routine X-ray examinations in English hospitals. National Radiological Protection Board. NRPB-R200, 1986.

4. International Commission on Radiological Protection (ICRP). 1990 Recommendations of the International Commission on Radiological Protection. ICRP publication 60. Ann ICRP. 1991;21(1-3):1-201.

5. International Commission on Radiological Protection (ICRP). Radiological Protection and Safety in Medicine. ICRP publication 73. Ann ICRP. 1996;26(2):1-47.

6. International Commission on Radiological Protection (ICRP). The 2007 Recommendations of the International Commission on Radiological Protection. ICRP publication 103. Ann ICRP. 2007;37(2-4):1-332.
7. International Commission on Radiological Protection (ICRP). Diagnostic reference levels of medical imaging. ICRP publication 135. Ann ICRP. 2017;46(1):1-143.

8. International Atomic Energy Agency (IAEA). Radiation protection and safety of radiation sources: international basic safety standards. The IAEA in Austria, 2014. https://www-pub.iaea.org/ MTCD/Publications/PDF/Pub1578_web-57265295.pdf. Accessed July 5, 2020.

9. Vassileva J, Rehani M. Diagnostic reference levels. Am J Roentgenol. 2015;204:W1-W3.

10. Watanabe H, Ishii K, Hosono M, Imabayashi E, Abe K, Inubushi M, Ohno K, Magata Y, Ono K, Kikuchi K, Wagatsuma K, Takase T, Saito K, Takahashi Y. Report of a nationwide survey on actual administered radioactivities of radiopharmaceuticals for diagnostic reference levels in Japan. Ann Nucl Med. 2016;30:435-44.

11. The Japanese Society of Nuclear Medicine, Optimization Committee for Pediatric Nuclear Medicine Studies, Koizumi K, Masaki H, Matsuda H, Uchiyama M, Okuno M, Oguma E, Onuma H, Kanegawa K, Kanaya S, Kamiyama H, Karasawa K, Kitamura M, Kida T, Kono T, Kondo C, Sasaki M, Terada H, Nakanishi A, Hashimoto T, Hataya H, Hamano S-I, Hirono K, Fujita Y, Hoshino K, Yano M, Watanabe S. Japanese consensus guidelines for pediatric nuclear medicine. Ann Nucl Med. 2014;28:498-503.

12. J-RIME. National diagnostic reference levels in Japan (2020) Japan 2020 DRLs. https://www.radher.jp/J-RIME/report/DRL20 20_Engver.pdf. Accessed Aug 6, 2020.

13. Becker MD, Butler PF, Siam M, Gress DA, Ghesani M, Harkness BA, Yoo DC, Oates MEUS. PET/CT and gamma camera diagnostic reference levels and achievable administered activities for noncardiac nuclear medicine studies. Radiology. 2019;293:203-11.

14. European Commission. Radiation protection no. 180. Diagnostic reference levels in thirty-six European Countries 2/2. European Union, 2014. https://ec.europa.eu/energy/sites/ener/files/docum ents/RP180\%2520part2.pdf. Accessed Aug 6, 2020.

15. Iball GR, Bebbington NA, Burniston M, Edyvean S, Fraser L, Julyan P, Parkar N, Wood T. A national survey of computed tomography doses in hybrid PET-CT and SPECT-CT examinations in the UK. Nucl Med Commun. 2017;38:459-70.

16. Gardner M, Katsidzira NM, Ross E, Larkin EA. Patient dosimetry audit for establishing local diagnostic reference levels for nuclear medicine CT. Br J Radiol. 2017;90:2160850.

17. Alkhybari EM, McEntee MF, Brennan PC, Willowson KP, Hogg $\mathrm{P}$, Kench PL. Determining and updating PET/CT and SPECT/ CT diagnostic reference levels: a systematic review. Radiat Prot Dosim. 2018;182:532-45.

18. Alessio AM, Farrell MB, Fahey FH. Role of reference levels in nuclear medicine: a report of the SNMMI Dose Optimization Task Force. J Nucl Med. 2015;56:1960-4.

19. Rehani MM. Limitations of diagnostic reference level (DRL) and introduction of acceptable quality dose (AQD). Br J Radiol. 2015;88:20140344.

Publisher's Note Springer Nature remains neutral with regard to jurisdictional claims in published maps and institutional affiliations. 


\section{Affiliations}

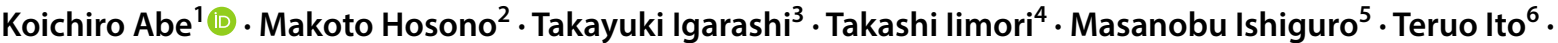 Tomomasa Nagahata $^{7} \cdot$ Hiroyuki Tsushima $^{8} \cdot$ Hiroshi Watanabe $^{9}$}

1 Department of Radiology, Tokyo Medical University, 6-7-1 Nishishinjuku, Shinjuku-ku, Tokyo 160-0023, Japan

2 Department of Radiology, Faculty of Medicine, Kindai University, 377-2 Ohno-Higashi, Osaka-Sayama,

Osaka 589-8511, Japan

3 Department of Radiological Technology, International University of Health and Welfare Narita Hospital, 852 Hatakeda, Narita-shi, Chiba 286-8520, Japan

4 Department of Radiological Technology, Chiba University Hospital, 1-8-1 Inohana, Chuo-ku, Chiba-shi, Chiba 260-8677, Japan

5 Department of Radiological Technology, Fujita Health University, 1-98 Dengakugakubo, Kutsukake-cho, Toyoake, Aichi 470-1192, Japan
6 Department of Radiological Sciences, International University of Health and Welfare, 4-3 Kozunomori, Narita-shi, Chiba 286-8686, Japan

7 Department of Radiological Technology, Osaka City University Hospital, 1-5-7, Asahi-machi, Abeno-ku, Osaka-shi, Osaka 545-8586, Japan

8 Department of Radiological Sciences, Ibaraki Prefectural University of Health Sciences, 4669-2 Ami, Ami-machi, Inashiki-gun, Ibaraki 300-0394, Japan

9 Department of Radiological Sciences, Faculty of Health Sciences, Gunma Paz University, 1-7-1 Tonyamachi, Takasaki-shi, Gunma 370-0006, Japan 